\title{
Changes in body mass index in children and adolescents in Israel during the COVID-19 pandemic
}

\author{
Shlomit Shalitin $\mathbb{D}^{1,2}{ }^{凶}$, Moshe Phillip ${ }^{1,2}$ and Michal Yackobovitch-Gavan ${ }^{1,2}$ \\ (c) The Author(s), under exclusive licence to Springer Nature Limited 2022
}

BACKGROUND: The current coronavirus disease 2019 (COVID-19) pandemic has health, social, and economic implications. Our primary objective was to evaluate changes in body mass index (BMI) from the pre-pandemic to COVID-19 pandemic period among a large pediatric population in Israel.

METHODS: This retrospective cohort study is based on data from Clalit Health Services, the largest health maintenance organization in Israel. The data accessed included sociodemographic, anthropometric, and clinical parameters of persons aged 2-20 years with at least one BMI measurement during 2017-2019 (pre-pandemic period) and one between April 1, 2020 and December 31, 2020 (pandemic period).

RESULTS: The cohort comprised 36,837 individuals (50.8\% females); median age 11.2 years, $83.6 \%$ were Jewish and $10.3 \%$ of Arab ethnicity. BMI-SDS increased in both sexes $(p<0.001)$, in both ethnicities $(p<0.001)$, in all socioeconomic position clusters $(p<0.001)$, in children aged $2-18$ years $(P<0.001)$, and in children with underweight or normal-weight in the pre-pandemic period $(p<0.001)$. For 21,610 individuals $(35.6 \%)$, BMI-SDS increased $\geq 0.25$ SD. The increase in BMI-SDS was greater in children aged $2-6$ compared to 6.1-18 years; BMI-SDS decreased among those aged 18.1-20 years $(P<0.001)$. The increase in BMI-SDS was greater among those with underweight than normal weight; BMI-SDS decreased among those with overweight and obesity $(P<0.001)$. During the pandemic, overweight or obesity presented in $11.2 \%$ of those with normal weight in the pre-pandemic period; and obesity presented in $21.4 \%$ of those with overweight in the pre-pandemic period.

CONCLUSIONS: The COVID-19 pandemic correlated with overall weight gain among children and adolescents, with the most substantial weight gain in children aged 2-6 years. Notably, the most significant increase in BMI-SDS was observed in children with underweight; BMI-SDS decreased in children with overweight and obesity. Policies should be established during the pandemic that focus on increasing physical activity, reducing sedentary time, and promoting healthy diets.

International Journal of Obesity (2022) 46:1160-1167; https://doi.org/10.1038/s41366-022-01092-5

\section{INTRODUCTION}

Obesity is a global public health challenge with worldwide increased incidence. Childhood obesity has reached pandemic proportions and is currently one of the most prevalent public health problems [1]. The pathophysiology of excess weight gain is complex, and includes interactions between genetic, biological, behavioral (physical inactivity, increased screen time), and socioeconomic factors [2-4].

The increasing prevalence of childhood obesity is associated with the emergence of comorbidities previously considered to be "adult" diseases, including obstructive sleep apnea (OSA), hypertension, dyslipidemia, type 2 diabetes, and non-alcoholic fatty liver disease (NAFLD) [5]. Children with obesity are likely to become adults with obesity; the latter confers increased risks of morbidity and mortality [6]. Therefore, controlling the childhood obesity pandemic has become a top public health priority worldwide.

The current coronavirus disease 2019 (COVID-19) pandemic has health, social, and economic implications. The protective and control measures that have been instituted by governments around the world include major lifestyle changes such as lockdowns and stay-at-home orders, and physical distancing. Schools and kindergartens have been closed and some children have lost the safety net of access to nutritious food, a safe place to be, and mandatory physical activity, as well as their social networks and regular routines.

The environments of nurseries and schools generally provide structure and routine around meal routines, physical activity, and sleep schedules, which are predominant lifestyle factors implicated in obesity risk. Accordingly, during non-pandemic periods, BMI gain was shown to be more rapid during summer vacation than during the "in-session" school year $[7,8]$.

Behaviors associated with increased weight gain such as sedentary behavior, increased screen time, poor diet, and irregular sleep are beneficially regulated when children follow a structured day. The difficulty to maintain a structured day for children during the COVID-19 pandemic, together with requirements for online classes, resulted in irregular sleep patterns and extensively prolonged screen times $[9,10]$. During non-pandemic periods, these conditions were found to be associated with weight gain

${ }^{1}$ The Jesse Z. and Sara Lea Shafer Institute of Endocrinology and Diabetes, National Center for Childhood Diabetes Schneider Children's Medical Center of Israel, Petah Tikva, Israel. ${ }^{2}$ Sackler Faculty of Medicine, Tel Aviv University, Tel Aviv, Israel. ${ }^{\varpi}$ email: shalitin@netvision.net.il

Received: 10 December 2021 Revised: 31 January 2022 Accepted: 2 February 2022

Published online: 16 February 2022 
and reduced fitness levels [11]. Lockdowns during the pandemic may also be conducive to less favorable diets [12], including higher consumption of highly processed food with high content of saturated fat, sugar, and salt. Increased intake of potato chips, red meat, and sugary drinks during lockdown periods have also been described $[13,14]$.

The unfamiliar and prolonged stress of quarantine may have a negative psychological impact on the child and family [15]. This could further promote unhealthy food intake through stressrelated eating, thereby leading to obesity [16]. Furthermore, financial losses and even existential threats consequent to the pandemic may promote difficulties for families to afford healthy food, which is usually more expensive.

From another perspective, restrictions during the lockdown limited restaurant and fast-food dining, and encouraged homecooked meals, which are generally healthier and with reduced caloric intake. Moreover, during the pandemic the accessibility and popularity of at-home physical activity increased, through use of various digital platforms and web-based applications [17]. Indeed, a few studies reported lower proportions of children with overweight after school closure periods [18], and stability of body composition parameters in persons with overweight and obesity [19]. These determinants of health may have impacted pediatric body weight during the first year of the COVID-19 pandemic.

The main aim of this retrospective study was to analyze changes in body mass index (BMI) among a large population of children, adolescents, and young adults during the COVID-19 pandemic in Israel. A secondary aim was to evaluate the reported prevalence of obesity-related comorbidities in this population.

\section{METHODS AND POPULATION Study design}

This observational population-based cohort study utilized the computerized clinical database of Clalit Health Services (CHS), which insures and provides medical services to $54 \%$ of the population of Israel. The comprehensive state-of-the-art computerized data warehouse of CHS aggregates the vast majority of individual data on medical service use and can be queried down to this level. Data are accumulated by continuous real-time input from physicians and health service providers, and include anthropometric measurements, laboratory data, and pharmaceutical information in addition to diagnoses. Diagnoses of chronic diseases are validated by systematic methodology based on the diagnosis of the primary care physician, chronic medication use, laboratory results, hospitalization diagnosis, and malignancy registry. CHS performs logistic checks by comparing diagnoses from various sources and by direct validation of the diagnoses by the treating physicians of each patient.

This study included data of individuals from three of the nine CHS districts in Israel, which together provide care for a diverse population of nearly 508,000 persons aged 2-20 years. We evaluated changes in BMI of this age group between the prepandemic and pandemic periods.

The study was approved by the local institutional review board. Subject consent was waived due to the retrospective design, based on data from medical records.

\section{Study population}

Study inclusion criteria were available data of at least one measurement of height and weight during 2017-2019 (prepandemic period) and at least one of each during the period from April 1 to December 312020 (the pandemic period). Notably, the year 2020 included three lockdown periods in Israel: March-May, September-October, and December. In addition, during most of this year (2020), kindergarten and schools were closed or functioned only partially, and social distancing was recommended.
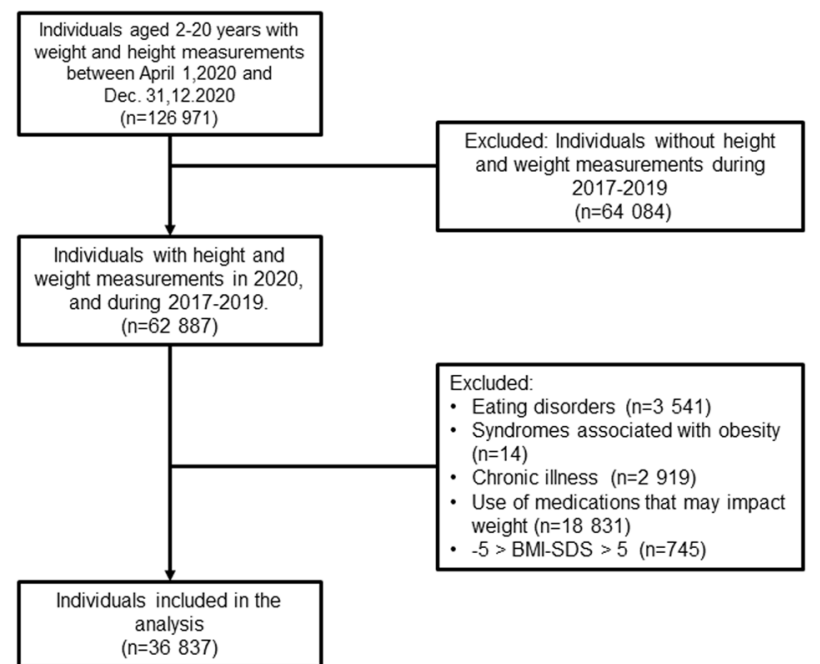

Fig. 1 Study flow chart of the participants inclusion. $n$ represents the number of individuals in each group. BMI-SDS body mass indexstandard deviation scores.

Conditions specifically associated with obesity were exclusion criteria. These included: genetic syndromes (such as Prader Willi syndrome and Alstrom syndrome), monogenetic obesity, and endocrine disorders (Cushing syndrome, untreated hypothyroidism, craniopharyngioma). The performance of bariatric surgery during the study period and the use of medications that may impact weight (oral/systemic steroids, methylphenidate and its derivate, anti-psychotic medications) were also exclusion criteria. Individuals with malignancies, chronic illness (type 1 diabetes, chronic renal failure, chronic heart disease, inflammatory bowel disease, cystic fibrosis, post solid organ transplantation treated with steroids), and eating disorders were not included. Those with improbable measurements $(-5>$ BMI-SDS $>5)$ were also excluded from the analysis.

Figure 1 shows the flow chart of the individuals included in the study.

\section{Data measurements and variables}

The following data were collected from the medical files of the individuals included: demographic parameters (sex, ethnicity, age), socioeconomic position (SEP), anthropometric parameters (height and weight as measured by nurses or pediatric physicians), and clinical parameters. The latter included the use of concomitant medications, the presence of concomitant illness, and the presence of documented obesity comorbidities. Examples of such comorbidities are dyslipidemia, type 2 diabetes or impaired glucose tolerance, hypertension, OSA, NAFLD, pseudotumor cerebri, slipped capital femoral epiphysis, and the presence of polycystic ovaries disease in adolescent females.

The SEP index of the Israel Central Bureau of Statistics is an adjusted calculation of 14 variables that measure social and economic levels in the domains of demographics, education, standard of living, and employment. SEP by home address was based on classification of towns and of neighborhoods in cities according to the socioeconomic level of the population in 2015 [20]. Israel Central Bureau of Statistics' SEP clusters are ranked on a scale of $1-10$, with 1 representing the lowest SEP. In the database of CHS, every 2 Israel Central Bureau of Statistics' SEP clusters are combined, for a total of five clusters. Accordingly, in CHS, SEP cluster 1 is defined as very low SEP, cluster 2 as low SEP, cluster 3 as intermediate SEP, cluster 4 as high SEP, and cluster 5 as very high SEP. These five clusters reflect Israel Central Bureau of Statistics' SEP levels of 1 and 2, 3 and 4, 5 and 6, 7 and 8, and 9 and 10 , respectively. 
Height was expressed as the standard deviation score (Ht-SDS) for age and sex. Body mass index (BMI) was calculated as weight (in kilograms) divided by height (in meters) squared. To compare BMI values across age groups by sex, BMI-standard deviation scores (SDSs) were calculated using the growth chart percentiles of the Centers for Disease Control and Prevention [21].

The study cohort was divided into three main age groups: preschool (ages 2-6 years), school age (ages 6.1-18 years), and young adults (ages 18.1-20 years).

Weight status was categorized as underweight for BMl values $<5$ th percentile (BMI-SDS $<-1.645$ ), normal for BMI between the 5 th and 84th percentiles $(-1.645 \leq$ BMI-SDS $\leq 1.036)$, overweight for BMI between the 85th and 95th percentiles $(1.036<$ BMI-SDS $\leq$ 1.645), and obese for $\mathrm{BMI}$ equal to or higher than the 95th percentile (BMI-SDS > 1.645).

We further analyzed changes in BMI-SDS by sex, ethnicity, age groups, and SEP category clusters; and also compared to prepandemic (years 2017-2019) BMI categories.

\section{Statistical analysis}

Analyses were carried out using SPSS, version 26.0 (IBM Corp, New York, NY, USA). Continuous variables with normal distributions are reported as means $\pm S D$, continuous variables with skewed distributions are reported as medians and inter-quartile ranges, and categorical variables are reported by numbers and percentages.

Differences in continuous data between males and females were examined using independent sample $t$-tests for normally distributed data or Mann-Whitney tests for skewed data, as appropriate. Pearson's Chi-square tests were used to examine differences in categorical data. One-way ANOVA and post-hoc Tukey were performed to compare changes in BMI-SDS between three or more categories.

Mixed-model repeated-measures analyses were conducted to evaluate the overtime trends in BMI-SDS (between the prepandemic years of 2017-2019 and the pandemic year of 2020) according to SEP parameters and BMI categories. The mixedmodel analysis enabled using all available data from the full cohort without imputing missing values. The models were specified with a within-group factor of time (the year of BMISDS evaluation), a between-group factor (according to sex, ethnicity, age category, SEP status, and BMI category) and the interaction of group with time. The data are expressed as estimated marginal means and standard errors.

To further explore if the change between 2019 and 2020 was a result of the pandemic or a general longitudinal annual trend, an additional sensitivity analysis was performed using mixed model repeated-measures analyses of two sub-cohorts, for the trends in BMI-SDS between 2017 and 2019 (pre-pandemic years only) and between 2018 and 2020 (including a pandemic year). Each analysis comprised a sub-cohort of the children aged 2-17 years at the baseline year (2017 or 2018). Two models for each period were performed. The first model included only a within-group factor of time (the year of BMISDS evaluation). The second model included, in addition, a between-group factor of BMI category and the interaction of BMI category with time.

Statistical significance was set at $P \leq 0.05$.

\section{RESULTS}

Characteristics of the study cohort are presented in Table 1. The cohort comprised 36,837 individuals (51.3\% females); $83.6 \%$ were of Jewish ethnicity and $10.3 \%$ of Arab ethnicity. The median age was 11.2 years.

In the pre-pandemic period, $13.2 \%$ were with overweight and $10.5 \%$ with obesity. During the pandemic period, $13.3 \%$ were with overweight and $12.3 \%$ with obesity $(P<0.001)$.
Table 2 presents the mixed model analyses for the trends in BMI-SDS from 2017 to 2019 (pre-pandemic period) to 2020 (pandemic period), adjusted by sex, age category, ethnicity, SEP category, and the BMI category in the pre-pandemic period. During the entire study period, females had higher BMI-SDS than males $\left(P_{\text {group }}<0.001\right)$. During the pandemic year, the BMI-SDS increased significantly compared to the pre-pandemic period in both males and females $\left(P_{\text {time }}<0.001\right)$. Throughout the entire study period, the BMI-SDS was lower in the youngest age group (age 2-6 years) than in the other age groups $\left(P_{\text {group }}<0.001\right)$. The trends (slopes) of change in BMI-SDS differed across the age categories $\left(P_{\text {interaction }}<0.001\right)$. In the pandemic compared to the pre-pandemic period, the increase in BMI-SDS was the greatest in children aged 2-6, and BMI-SDS decreased among those aged 18.1-20 years. During the entire study period, the BMI-SDS was higher among those with Arab than Jewish ethnicity $\left(P_{\text {group }}<\right.$ $0.001)$. BMI-SDS increased significantly during the pandemic year compared to the pre-pandemic period in both ethnicities $\left(P_{\text {time }}<\right.$ $0.001)$. Also, during the entire study period, BMI-SDS was higher among those in the two lowest compared to the two highest SEP clusters $\left(P_{\text {group }}<0.001\right)$. However, during the pandemic year, the BMI-SDS increased significantly compared to the pre-pandemic period in all SEP clusters $\left(P_{\text {time }}<0.001\right)$. BMI-SDS increased significantly in the pandemic period, among children with underweight or normal weight in the pre-pandemic years, and decreased among those with overweight or obesity $\left(P_{\text {interaction }}<\right.$ 0.001).

Our sub-cohort analysis showed that the annual increases in BMI-SDS in 2018 and 2019 were lower $(<0.016$ SD) than the annual increases in 2019 and 2020 (0.031 SD) (supplementary Table 1). The increase in BMI-SDS was significant in the analysis that included the pandemic year $\left(P_{\text {time }}<0.001\right.$ and $\left.P_{\text {interaction }}<0.001\right)$ but not in the analysis that entailed only the pre-pandemic period $\left(P_{\text {time }}=0.131\right.$ and $\left.P_{\text {interaction }}=0.250\right)$. The most significant increase in BMI-SDS was observed among those with underweight at baseline (no significant change between 2017 and 2019, and an increase of 0.662 SD between 2019 and 2020). BMI-SDS decreased significantly between 2019 and 2020 among those with overweight and obesity at baseline $(-0.160$ SD and -0.188 SD, respectively) (supplementary Table 1 ).

Of the 3245 individuals with underweight in the pre-pandemic period, $1524(47.0 \%)$ were with normal weight during the pandemic, $26(0.8 \%)$ with overweight, and $16(0.5 \%)$ with obesity. Of the 24,867 individuals with normal weight in the pre-pandemic period, $1456(5.9 \%)$ were with underweight in the pandemic period, 2119 (8.5\%) with overweight, and 677 (2.7\%) with obesity. Of the 4861 with overweight in the pre-pandemic period, 14 $(0.3 \%)$ were with underweight in the pandemic period, 1770 (36.4\%) with normal weight, and 1041 (21.4\%) with obesity. Of the 3864 individuals with obesity in the pre-pandemic period, $5(0.1 \%)$ were with underweight in the pandemic period, 335 (8.7\%) with normal weight, and 730 (18.9\%) with overweight.

Table 3 shows crude changes in BMI-SDS and the proportions of individuals with increases in BMI-SDS $\geq 0.25$ and $\geq 1$ during the pandemic period, stratified by sex, age category, ethnicity, SEP category, and the BMI category in the pre-pandemic period. The mean change in BMI-SDS during the pandemic period was $0.055 \pm 0.84$. Supporting the mixed model analysis, a significantly greater increase in BMI-SDS was observed in children aged 2-6 compared to 6.1-18 years, and a decrease in BMI-SDS was observed in the 18.1-20-year group $(P<0.001)$. The increase in BMI-SDS was also greater in individuals with underweight compared to normal weight, and a decrease in BMI-SDS was observed in those with overweight and obesity $(P<0.001)$. The increase in BMI-SDS was comparable between males and females, between those of Jewish and Arab ethnicities, and between SEP categories. In 13,125 individuals (35.6\% of the study cohort), BMISDS increased by $\geq 0.25$; and in 3861 (10.5\% of the cohort), BMI- 
Table 1. Demographic and anthropometric characteristics of the study cohort.

\begin{tabular}{|c|c|c|c|c|}
\hline & All $n=36,837$ & Males $n=17,934(48.7 \%)$ & Females $n=18,903(51.3 \%)$ & $P$ \\
\hline \multicolumn{5}{|c|}{ Age in 2020 (years) } \\
\hline Median (IQR) & $11.2(6.6,16.1)$ & $11.1(6.3,16.1)$ & $11.3(6.8,16.1)$ & $<0.001$ \\
\hline \multicolumn{5}{|l|}{ Age group $n(\%)$} \\
\hline $2-6$ years & $10,138(27.6 \%)$ & $5248(29.3 \%)$ & 4890 (25.9\%) & $<0.001$ \\
\hline $6.1-18$ years & $23,849(64.7 \%)$ & $11,369(63.4 \%)$ & $12,480(66.0 \%)$ & \\
\hline $18.1-20$ years & $2850(7.7 \%)$ & $1317(7.3 \%)$ & $1533(8.1 \%)$ & \\
\hline Jewish & $30,766(83.5 \%)$ & 14,999 (83.6\%) & $15,777(83.5 \%)$ & 0.005 \\
\hline Arab & $3782(10.3 \%)$ & 1772 (9.9\%) & $2010(10.6 \%)$ & \\
\hline Unknown & $2279(6.2 \%)$ & $1163(6.5 \%)$ & $1116(5.9 \%)$ & \\
\hline \multicolumn{5}{|c|}{ SEP category (range 1-5) } \\
\hline Median (IQR) & $2(2,3)$ & $2(2,3)$ & $2(2,3)$ & 0.207 \\
\hline \multicolumn{5}{|l|}{ SEP (\%) } \\
\hline Low & $6991(19.0 \%)$ & 3345 (18.7\%) & $3646(19.3 \%)$ & \\
\hline Very low & $357(1.0 \%)$ & $175(1.0 \%)$ & $182(1.0 \%)$ & \\
\hline Unknown & $1221(3.3 \%)$ & $581(3.2 \%)$ & $1047(3.4 \%)$ & \\
\hline \multicolumn{5}{|c|}{ Height-SDS pre-pandemic (2017-2019) } \\
\hline Mean (SD) & $-0.093 \pm 1.106$ & $-0.099 \pm 1.124$ & $-0.087 \pm 1.090$ & 0.284 \\
\hline \multicolumn{5}{|l|}{ Height-SDS 2020} \\
\hline Mean (SD) & $-0.092 \pm 1.108$ & $-0.092 \pm 1.119$ & $-0.091 \pm 1.097$ & 0.961 \\
\hline \multicolumn{5}{|c|}{ BMI-SDS pre-pandemic (2017-2019) } \\
\hline Mean (SD) & $0.083 \pm 1.254$ & $0.009 \pm 1.308$ & $0.152 \pm 1.196$ & $<0.001$ \\
\hline \multicolumn{5}{|c|}{ BMI category pre-pandemic (2017-2019) } \\
\hline \multicolumn{5}{|c|}{ BMI category 2020} \\
\hline \multicolumn{5}{|l|}{$n(\%)$} \\
\hline Underweight & $3154(8.6 \%)$ & $1825(10.2 \%)$ & $1329(7.0 \%)$ & $<0.001$ \\
\hline Normal weight & $24,244(65.8 \%)$ & $11,697(65.2 \%)$ & $12,547(66.4 \%)$ & \\
\hline Overweight & $4911(13.3 \%)$ & $2125(11.8 \%)$ & $2786(14.7 \%)$ & \\
\hline Obesity & 4528 (12.3\%) & 2287 (12.8\%) & 2241 (11.9\%) & \\
\hline
\end{tabular}

Data are presented as means \pm SD (normal distribution), median and interquartile range (skewed distribution), or number and percent (categorial variable). $P$ values represent independent samples $t$-test or Mann-Whitney U-test, for variables with normal or skewed distribution, respectively; or Pearson's chi-square test for categorial variables.

BMI-SDS categories: underweight BMI-SDS $<-1.645$, normal weight $-1.645 \leq$ BMI-SDS $\leq 1.036$, overweight $1.036<$ BMI-SDS $\leq 1.645$, obesity BMI-SDS > 1.645 SEP socioeconomic position. BMI-SDS body mass index standard deviation score.

SDS increased by $\geq 1.0$. The proportions of individuals with increases in BMI-SDS $\geq 0.25$ and $\geq 1.0$ during the year of the pandemic were significantly higher among males than females $(p<0.001)$, among children aged $2-6$ years compared to the older age groups $(p<0.001)$, and among those with underweight compared to overweight and obesity in the pre-pandemic years $(p<0.001)$. The proportion of individuals with an increase in BMISDS $\geq 0.25$ only (not $\geq 1.0$ ), was also significantly higher among the individuals of Jewish compared to Arab ethnicity $(p<0.001)$, and among those from the two high SEP clusters compared to the other SEP clusters $(p<0.001)$.

Table 4 presents the reported prevalence of obesity-related comorbidities of the study cohort in males and females. Table 5 presents such prevalence according to weight status categories in 2020. The prevalence of OSA and hypertension were significantly higher in males $(p<0.001)$, whereas the prevalence of pseudotumor cerebri and dyslipidemia were significantly higher in females $(p=0.032$ and $p=0.037$, respectively, Table 4$)$. 
Table 2. Mixed model repeated measures analyses for the trends in BMI-SDS from 2017 to 2020.

\begin{tabular}{|c|c|c|c|c|c|c|c|}
\hline Comparison parameter & BMI-SDS 2017 & BMI-SDS 2018 & BMI-SDS 2019 & BMI-SDS 2020 & $P$ time & $P$ group & $P$ time $X$ group \\
\hline All & $0.075^{\mathrm{a}}(0.007)$ & $0.095^{\mathrm{b}}(0.008)$ & $0.097^{b}(0.008)$ & $0.138^{\mathrm{c}}(0.007)$ & $<0.001$ & & \\
\hline \multicolumn{8}{|l|}{ Sex } \\
\hline Males & $0.003^{\mathrm{a}}(0.011)$ & $0.024^{\mathrm{a}}(0.013)$ & $0.019^{\mathrm{a}}(0.012)$ & $0.068^{\mathrm{b}}(0.010)$ & $<0.001$ & $<0.001$ & 0.618 \\
\hline \multicolumn{8}{|l|}{ Age category } \\
\hline $2-6$ years & $-0.214^{\mathrm{a}}(0.016)$ & $-0.165^{\mathrm{b}}(0.017)$ & $-0.141^{\mathrm{b}}(0.015)$ & $-0.029^{c}(0.013)$ & 0.113 & $<0.001$ & $<0.001$ \\
\hline \multicolumn{8}{|l|}{ Ethnicity } \\
\hline Jewish & $0.040^{\mathrm{a}}(0.008)$ & $0.061^{\mathrm{b}}(0.009)$ & $0.066^{b}(0.008)$ & $0.105^{c}(0.007)$ & $<0.001$ & $<0.001$ & 0.192 \\
\hline Arab & $0.445^{\mathrm{a}}(0.024)$ & $0.500^{\mathrm{b}}(0.027)$ & $0.448^{\mathrm{a}}(0.025)$ & $0.518^{\mathrm{b}}(0.022)$ & & & \\
\hline \multicolumn{8}{|l|}{ SEP category } \\
\hline Underweight & $-2.333^{\mathrm{a}}(0.016)$ & $-2.298^{\mathrm{a}}(0.023)$ & $-2.351^{\mathrm{a}}(0.022)$ & $-1.656^{\mathrm{b}}(0.020)$ & $<0.001$ & $<0.001$ & $<0.001$ \\
\hline Normal weight & $-0.163^{\mathrm{a}}(0.006)$ & $-0.148^{\mathrm{ab}}(0.008)$ & $-0.135^{\mathrm{b}}(0.007)$ & $-0.099^{c}(0.006)$ & & & \\
\hline Overweight & $1.321^{\mathrm{a}}(0.004)$ & $1.333^{\mathrm{a}}(0.007)$ & $1.321^{\mathrm{a}}(0.007)$ & $1.168^{\mathrm{b}}(0.009)$ & & & \\
\hline Obesity & $2.063^{\mathrm{a}}(0.008)$ & $2.083^{\mathrm{ab}}(0.011)$ & $2.090^{\mathrm{b}}(0.010)$ & $1.877^{\mathrm{c}}(0.010)$ & & & \\
\hline
\end{tabular}

Table 3. Changes in BMI-SDS and the proportions of individuals with increases in BMI-SDS $\geq 0.25$ and $\geq 1$ during the year of the pandemic.

\begin{tabular}{|c|c|c|c|c|c|c|c|}
\hline Comparison parameter & n 36,837 & $\Delta \mathrm{BMI}-\mathrm{SDS}($ mean $\pm \mathrm{SD})$ & $P^{1}$ & $\Delta$ BMI-SDS $\geq 0.25 n(\%)$ & $P^{2}$ & $\Delta$ BMI-SDS $\geq 1.0 n(\%)$ & $P^{3}$ \\
\hline \multicolumn{8}{|l|}{ Sex } \\
\hline Males & 17,934 & $0.059 \pm 0.89$ & 0.419 & $6548(36.5 \%)$ & 0.001 & $2080(11.6 \%)$ & $<0.001$ \\
\hline \multicolumn{8}{|l|}{ Age category } \\
\hline $2-6$ years & 10,138 & $0.146 \pm 1.02^{\mathrm{a}}$ & $<0.001$ & 4502 (44.4\%) & $<0.001$ & $1744(17.2 \%)$ & $<0.001$ \\
\hline $6.1-18$ years & 23,849 & $0.043 \pm 0.77^{b}$ & & 8055 (33.8\%) & & $2010(8.4 \%)$ & \\
\hline \multicolumn{8}{|l|}{ Ethnicity } \\
\hline Jewish & 30,776 & $0.056 \pm 0.83$ & 0.599 & $11,062(35.9 \%)$ & $<0.001$ & $3238(10.5 \%)$ & 0.546 \\
\hline Arab & 3782 & $0.063 \pm 0.87$ & & 1244 (32.9\%) & & $410(10.8 \%)$ & \\
\hline \multicolumn{8}{|l|}{ SEP category } \\
\hline High and very high & 17,815 & $0.064 \pm 0.82$ & 0.281 & $6503(36.5 \%)$ & 0.002 & $1899(10.7 \%)$ & 0.742 \\
\hline Normal weight & 24,867 & $0.053 \pm 0.81^{b}$ & & 9373 (37.7\%) & & $2629(10.6 \%)$ & \\
\hline Overweight & 4861 & $-0.156 \pm 0.64^{c}$ & & $1193(24.5 \%)$ & & $93(1.9 \%)$ & \\
\hline Obesity & 3864 & $-0.201 \pm 0.60^{d}$ & & 535 (13.8\%) & & $11(0.3 \%)$ & \\
\hline
\end{tabular}

SEP socioeconomic position.

$\triangle \mathrm{BMI}-\mathrm{SDS}=\mathrm{BMI}-\mathrm{SDS}$ in 2020 minus the mean BMI-SDS in the pre-pandemic period.

$P^{1}$ represents the difference in $\triangle B M I-S D S$ between categories using 1-way ANOVA and post-hoc Tukey. Rates with different superscripts (a, b, $c$, d) differ significantly from each other at $P \leq 0.05$; rates with no superscripts do not differ significantly from each other.

$P^{2}$ and $P^{3}$ represent differences in the proportions of individuals with $\triangle \mathrm{BMI}-\mathrm{SDS} \geq 0.25$ and $\triangle \mathrm{BMI}-\mathrm{SDS} \geq 1.0$, respectively, between categories, using Pearson's Chi-square tests.

BMI-SDS body mass index standard deviation score. BMI-SDS categories: underweight BMI-SDS $<-1.645$, normal weight $-1.645 \leq$ BMI-SDS $\leq 1.036$, overweight $1.036<$ BMI-SDS $\leq 1.645$, obesity BMI-SDS $>1.645$. 
The rates of type 2 diabetes/impaired glucose tolerance, OSA, pseudotumor cerebri, hypertension, dyslipidemia, and NAFLD were higher among those with overweight and obesity than those with normal weight and underweight. The highest prevalence of obesity-related comorbidities was among those with obesity $(P<$ 0.001 for all comparisons, Table 5).

\section{DISCUSSION}

We report that BMI-SDS increased significantly in children and adolescents during the pandemic period. The general longitudinal annual trend alone does not explain this observation. The overall prevalence of obesity increased by $1.8 \%$. In our cohort, $11.2 \%$ of those who were of normal weight in the pre-pandemic period were with overweight or obesity during the pandemic, and $21.4 \%$ of those with overweight in the pre-pandemic period were with obesity during the pandemic. This increase in BMI-SDS may be attributed to the pandemic-related lifestyle changes reported in previous literature, including decreased physical activity [22, 23], changes in diet composition [13, 24], and increased indoor sedentary behaviors; and the closure of nurseries and schools. The structured days that are usually provided by nurseries and schools exert a positive impact on children's weight-related behaviors (e.g., sleep, diet, physical activity, screen time). During the COVID19 pandemic, children's access to regularly scheduled, compulsory physical activity decreased. The lack of the school day likely led to irregular sleeping patterns with later sleep schedules [9]; such

Table 4. Reported obesity-related comorbidities.

\begin{tabular}{|llllr} 
& $\begin{array}{l}\text { All } \boldsymbol{n}= \\
\mathbf{3 6 , 8 3 7}\end{array}$ & $\begin{array}{l}\text { Males } \boldsymbol{n}= \\
\mathbf{1 7 , 9 3 4} \\
\mathbf{( 4 8 . 7 \% )}\end{array}$ & $\begin{array}{l}\text { Females } \boldsymbol{n} \\
=\mathbf{1 8 , 9 0 3} \\
\mathbf{( 5 1 . 3 \% )}\end{array}$ & $\boldsymbol{P}$ \\
\hline Type 2 DM/ IGT & $429(1.2 \%)$ & $220(1.2 \%)$ & $209(1.1 \%)$ & 0.279 \\
\hline OSA & $313(0.8 \%)$ & $188(1.0 \%)$ & $125(0.7 \%)$ & $<0.001$ \\
\hline PTC & $53(0.1 \%)$ & $18(0.1 \%)$ & $35(0.2 \%)$ & 0.032 \\
\hline Hypertension & $194(0.5 \%)$ & $125(0.7 \%)$ & $69(0.4 \%)$ & $<0.001$ \\
\hline Dyslipidemia & $549(1.5 \%)$ & $243(1.4 \%)$ & $306(1.6 \%)$ & 0.037 \\
\hline NAFLD & $189(0.5 \%)$ & $92(0.5 \%)$ & $97(0.5 \%)$ & 0.998 \\
\hline SCFE & 7 & 3 & 4 & 0.999 \\
& $(<0.001 \%)$ & $(<0.001 \%)$ & $(<0.001 \%)$ & \\
\hline PCO & 8 & & 8 & \\
& $(<0.001 \%)$ & & $(<0.001 \%)$ & \\
\hline
\end{tabular}

Data are presented as number (percent). $P$ values represent Pearson's chisquare tests.

$D M$ diabetes mellitus, IGT impaired glucose tolerance, OSA obstructive sleep apnea, PTC pseudotumor cerebri, NAFLD non-alcoholic fatty liver disease, SCFE slipped capital femoral epiphysis, PCO polycystic ovary syndrome. patterns have been linked to increased risk for overweight or obesity.

Our findings corroborate the 10 -fold increase in BMI-SDS reported during the same period as our study (2017-2020) among 1770 children from the southeastern US [25]. In that study, accelerated BMI-SDS gain was observed across children of different racial groups, grade levels, and in both boys and girls.

A number of studies have examined an association of child's sex with overweight and obesity. Some reported no sex-difference [26], some reported higher overweight prevalence among boys [27], whereas other reported higher overweight rates among girls [28]. In our cohort, BMI-SDS was higher in females than males in both the pre-pandemic and pandemic periods. During the prepandemic period, $24.3 \%$ of the females vs. $23.0 \%$ of the males were with overweight or obesity. During the pandemic, $26.6 \%$ of the females vs. $24.6 \%$ of the males were with overweight or obesity. The increase in BMI-SDS during the pandemic was similar for the two sexes. Yet, in a slightly greater proportion $(1.7 \%$ difference) of males, BMI-SDS increased by $\geq 0.25$. Our results might be explained by a more dramatic change in lifestyle during the lockdown for males, who generally tend to have a more active and less sedentary lifestyle than females. Our findings contrast to studies that reported significantly higher increases in BMI among males than females during the COVID-19 pandemic $[29,30]$.

We report higher BMI-SDS among individuals of Arab compared to Jewish ethnicity, both in the pre-pandemic and the pandemic periods. This ethnical difference was previously reported in a study conducted prior to the COVID-19 pandemic [31]. Notably, we found a comparable increase in BMI-SDS in both ethnicities during the pandemic.

The COVID-19 pandemic has led to an economic crisis, characterized by increasing rates of unemployment and financial insecurity. Coping with financial strain may negatively impact health behaviors, such as leading to unhealthy food choices and decreased physical activity. In our cohort, individuals from the low SEP clusters had higher BMI-SDS both in the pre-pandemic and pandemic periods. However, increases in BMI-SDS among SEP clusters were comparable, and increased BMI-SDS of $\geq 0.25$ was observed in even a higher proportion of those in the higher SEP clusters. This contrasts to a previous study [32] that showed a higher rate of weight gain during the COVID-19 pandemic among children of lower socioeconomic status. A possible explanation for our findings is that families from higher SEP clusters spent more time at home, during which they prepared more meals, with increased caloric intake. On the other hand, families from the lower SEP clusters had more financial issues with food insecurities.

Interestingly, we found that during the pandemic year, the change in BMI-SDS differed significantly between age groups. BMISDS increased significantly compared to the pre-pandemic period in children aged 2-6 years, as previously reported [29, 32]. While the increase in BMI-SDS was the greatest in children aged 2-6 compared to 6.1-18 years, it decreased among those aged 18.1-20 years. We assume that the youngest age group is more

Table 5. Prevalence of obesity-related comorbidities according to weight status categories in 2020.

\begin{tabular}{|c|c|c|c|c|c|}
\hline & Underweight $n=3154$ & Normal weight $n=24,244$ & Overweight $n=4911$ & Obesity $n=4528$ & $\boldsymbol{P}$ \\
\hline Type 2 DM/ IGT & $23(0.7 \%)$ & $261(1.1 \%)$ & $70(1.4 \%)$ & 75 (1.7\%) & $<0.001$ \\
\hline OSA & $17(0.5 \%)$ & $176(0.7 \%)$ & $40(0.8 \%)$ & $80(1.8 \%)$ & $<0.001$ \\
\hline PTC & $1(<0.001 \%)$ & $22(0.1 \%)$ & $7(0.1 \%)$ & $23(0.5 \%)$ & $<0.001$ \\
\hline Hypertension & $11(0.3 \%)$ & $85(0.4 \%)$ & $40(0.8 \%)$ & $58(1.3 \%)$ & $<0.001$ \\
\hline Dyslipidemia & $33(1.0 \%)$ & $309(1.3 \%)$ & $89(1.8 \%)$ & $118(2.6 \%)$ & $<0.001$ \\
\hline NAFLD & $4(0.1 \%)$ & $27(0.1 \%)$ & $37(0.8 \%)$ & $121(2.7 \%)$ & $<0.001$ \\
\hline
\end{tabular}

Data are presented as number (percent). $P$ values represent Pearson's chi-square tests.

DM diabetes mellitus, IGT impaired glucose tolerance, OSA obstructive sleep apnea, PTC pseudotumor cerebri, NAFLD non-alcoholic fatty liver disease. 
prone to intercurrent viral illnesses, with decreased food intake. However, during the pandemic, such viral illnesses were reduced due to social distancing and closing of daycare centers. Further, increased snacking and more meals prepared and consumed at home may have promoted weight gain. Nevertheless, this observation is very concerning and underscores the importance of obesity prevention and management efforts during and following the COVID-19 pandemic, including increased access to efforts that promote healthy behaviors.

We found that children with underweight or normal weight in the pre-pandemic period experienced higher acceleration in BMISDS change compared to children with overweight or obesity. Our findings corroborate those of Weaver et al. [25] and Azoulay et al. [19] who found that children with normal weight, but not those with overweight or obesity, experienced significant acceleration in BMI-SDS change. Our findings contrast to a longitudinal study that showed sharp increases in BMI rates during the COVID-19 pandemic among persons aged 2-19 years in the US, especially among those with overweight or obesity [33]. A possible explanation for our findings is that children with overweight or obesity may have already been engaging in risky obesogenic behaviors before the pandemic. Thus, behavioral changes were not as dramatic as for children with normal weight, and in turn, BMI gain did not increase as dramatically. However, because behavioral data were not collected, this is speculative.

Notably, weight status improved for the $47 \%$ of the individuals in our cohort who were with underweight in the pre-pandemic period and subsequently with normal weight during the pandemic. Moreover, $36 \%$ of those with overweight in the prepandemic period were with normal weight during the pandemic. Of those with obesity in the pre-pandemic period, $8.7 \%$ were with normal weight and $19 \%$ with overweight during the pandemic. A possible explanation for these findings is that in some families, more time at home may have changed family practices such as to dedicate greater time to planning and preparing meals, with more parental control of meal frequency and content. Other possible explanations for this observation may be related to public health messages that emphasized a role of excess weight in exacerbating risks of worse outcomes of COVID-19. Such messages might have encouraged adolescents and young adults with overweight and obesity to invest more effort to improve their weight status. Another possible explanation relates to the increased rate of eating disorders that was reported during the COVID-19 pandemic $[34,35]$. Although we excluded individuals with confirmed eating disorders, individuals with undiagnosed eating disorders may have been included, and this could have impacted weight status. Therefore, we summarize that the pandemic increased the general variability in BMI SDS change.

Rates of obesity-related comorbidities in our cohort were lower than previously published for the general pediatric ages [36-39]. This may be due to underreport. However, the rates of most of the investigated comorbidities were higher in those with overweight and obesity compared to those with normal weight and underweight; the highest rate was in individuals with obesity.

To the best of our knowledge, this is the first study to analyze weight changes in a very large population of children and adolescents during the COVID-19 pandemic in Israel. The strengths of our study include: the longitudinal evaluation of a cohort of children and adolescents from the pre-pandemic to the pandemic period, with the use of objective measurements of BMI, and representation of a large age group, different ethnicities, and different SEP clusters.

Yet, some limitations exist. First, our sample comprised only individuals residing in three districts in the center of the country. Therefore, the generalizability of this study may be limited. Nonetheless, the cohort included individuals of both Arab and Jewish ethnicity, and diverse SEP clusters. Second, only children and adolescents who attended clinics and had anthropometric measurements in the pre-pandemic period and during the pandemic period were included. Possibly the parents of those included were especially interested in their children's growth status. Therefore, the observed changes in BMI might have been underestimated compared to those for the general population. Also, the lack of information on diet and physical activity limits comparing outcomes between the pandemic and pre-pandemic periods.

In conclusion, in this large longitudinal cohort, the COVID-19 pandemic correlated with general weight gain among children and adolescents. Children younger than age 6 years showed the most substantial weight gain. Notably, despite the overall gain in BMI-SDS, we observed variability of BMI-SDS change among weight status categories. The most significant increase occurred among children with underweight; a significant decrease occurred in children with overweight and obesity. Policies and precautionary measures should be established during the pandemic, with a focus on increasing physical activity, reducing sedentary time, and promoting healthy diets.

\section{REFERENCES}

1. Di Cesare M, Sorić M, Bovet P, Miranda JJ, Bhutta Z, Stevens GA, et al. The epidemiological burden of obesity in childhood: a worldwide epidemic requiring urgent action. BMC Med. 2019;17:212.

2. Campbell MK. Biological, environmental and social influences on childhood obesity. Pediatr Res. 2016;20:205-11.

3. Ang YN, Wee BS, Poh BK, Ismail MN. Multifactorial Influences of Childhood Obesity. Curr Obes Rep. 2013;30:10-22.

4. Luiggi M, Rey O, Travert M, Griffet J. Overweight and obesity by school socioeconomic composition and adolescent socioeconomic status: a school-based study. BMC Public Health. 2021;11:1837.

5. Kumar S, Kelly AS. Review of childhood obesity: from epidemiology, etiology, and comorbidities to clinical assessment and treatment. Mayo Clin Proc. 2017;92:251-65.

6. Llewellyn A, Simmonds M, Owen CG, Woolacott N. Childhood obesity as a predictor of morbidity in adulthood: a systematic review and meta-analysis. Obes Rev. 2016;17:56-67.

7. Von Hippel PT, Powell B, Downey DB, Rowland NJ. The effect of school on overweight in childhood: Gain in body mass index during the school year and during summer vacation. Am J Public Health. 2007;97:696-702.

8. Franckle R, Adler R, Davison K. Accelerated weight gain among children during summer versus school year and related racial/ethnic disparities: A systematic review. Prev Chronic Dis. 2014;11:E101.

9. Guo YF, Liao MQ, Cai WL, Yu XX, Li SN, Ke XY, et al. Physical activity, screen exposure and sleep among students during the pandemic of COVID-19. Sci Rep. 2021:20:8529.

10. Nagata JM, Abdel Magid HS, Pettee Gabriel K. Screen time for children and adolescents during the coronavirus disease 2019 pandemic. Obesity. 2020;28:1582-3.

11. Fang $K$, Mu M, Liu K, He Y. Screen time and childhood overweight/obesity: A systematic review and meta-analysis. Child Care Health Dev. 2019;45:744-53.

12. Calcaterra V, Vandoni M, Pellino VC, Cena H. Special attention to diet and physical activity in children and adolescents with obesity during the coronavirus disease2019 pandemic. Front Pediatr. 2020;8:407.

13. Pietrobelli A, Pecoraro L, Ferruzzi A, Heo M, Faith $M$, Zoller $T$, et al. Effects of COVID-19 lockdown on lifestyle behaviors in children with obesity living in Verona, Italy: a longitudinal study. Obesity. 2020;28:1382-5.

14. Ruiz-Roso MB, de Carvalho Padilha $P$, Mantilla-Escalante DC, Ulloa N, Brun $P$, Acevedo-Correa D, et al. Covid-19 confinement and changes of adolescent's dietary trends in Italy, Spain, Chile, Colombia and Brazil. Nutrients. 2020;12:1807.

15. Liu JJ, Bao Y, Huang X, Shi J, Lu L. Mental health considerations for children quarantined because of COVID-19. Lancet Child Adolesc Health. 2020;4:347-9.

16. Rasheed N. Stress-associated eating leads to obesity. Int J Health Sci. 2017;11:1-2.

17. Parker K, Uddin R, Ridgers ND, Brown H, Veitch J, Salmon J, et al. The use of digital platforms for adults' and adolescents' physical activity during the COVID19 Pandemic (Our Life at Home): Survey Study. J Med Internet Res. 2021;1: e23389.

18. Wen J, Zhu L, Ji C. Changes in weight and height among Chinese preschool children during COVID-19 school closures. Int J Obes. 2021;45:2269-73.

19. Azoulay E, Yackobovitch-Gavan M, Yaacov H, Gilboa I, Lopez A, Sheppes T, et al. Weight status and body composition dynamics in children and adolescents during the COVID-19 pandemic. Front Pediatr. 2021;9:707773. 
20. Israel central bureau of statistics (CBS); 2019. Characterization and classification of geographical units by the socio-economic level of the population, 2015. https:// www.cbs.gov.il/he/publications/DocLib/2019/1765_socio_economic_2015/ e_print.pdf.

21. Kuczmarski RJ, Ogden CL, Guo SS, Grummer-Strawn LM, Flegal KM, Mei Z, et al. CDC growth charts for the United States: methods and development. Vital Health Stat. 2002;246:1-190.

22. Dunton GF, Do B, Wang SD. Early effects of the COVID-19 pandemic on physical activity and sedentary behavior in children living in the U.S. BMC Public Health. 2020;20:1351.

23. Jia P, Zhang L, Yu W, Yu B, Liu M, Zhang D, et al. Impact of COVID-19 lockdown on activity patterns and weight status among youths in China: the COVID-19 impact on lifestyle change survey (COINLICS). Int J Obes. 2021;45:695-9.

24. Adams EL, Caccavale LJ, Smith D, Bean MK. Food insecurity, the home food environment, and parent feeding practices in the era of COVID-19. Obesity. 2020;28:2056-63.

25. Weaver RG, Hunt ET, Armstrong B, Beets MW, Brazendale K, Turner-McGrievy G, et al. COVID-19 leads to accelerated increases in children's BMI z-Score gain: An interrupted time-series study. Am J Prev Med. 2021;61:e161-e169.

26. Baskin ML, Ard J, Franklin F, Allison DB. Prevalence of obesity in the United States. Obes Rev. 2005;6:5-7.

27. Hedley AA, Ogden CL, Johnson CL, Carroll MD, Curtin LR, Flegal KM. Prevalence of overweight and obesity among US children, adolescents, and adults, 1999-2002. JAMA. 2004;291:2847-50.

28. Salazar-Martinez E, Allen B, Fernandez-Ortega C, Torres-Mejia G, Galal O, LazcanoPonce E. Overweight and obesity status among adolescents from Mexico and Egypt. Arch Med Res. 2006;37:535-42.

29. Vinker-Shuster M, Grossman ES, Yeshayahu Y. Increased weight gain of children during the COVID-19 lockdown. Isr Med Assoc J. 2021;23:219-22.

30. Maltoni G, Zioutas M, Deiana G, Biserni GB, Pession A, Stefano, et al. Gender differences in weight gain during lockdown due to COVID-19 pandemic in adolescents with obesity. Nutr Metab Cardiovasc Dis. 2021;31:2181-5.

31. Ram E, Marcus O, Joubran S, Abdo B, Asal NR. Prevalence of obesity among Arab school children in Nazareth, Israel: comparison with national (Jewish) and international data. Pediatr Obes. 2013;8:428-38.

32. Jenssen BP, Kelly MK, Powell M, Bouchelle Z, Mayne SL, Fiks AG. COVID-19 and Changes in Child Obesity. Pediatrics. 2021;147:e2021050123.

33. Lange SJ, Kompaniyets L, Freedman DS, Kraus EM, Porter R, Blanck HM, et al. Longitudinal trends in body mass index before and during the COVID-19 pandemic among persons aged 2-19 years-United States, 2018- 2020. MMWR Morb Mortal Wkly Rep. 2021;70:1278-83.

34. Agostino H, Burstein B, Moubayed D, Taddeo D, Grady R, Vyver E, et al. Acute care visits for eating disorders among children and adolescents after the onset of the COVID-19 pandemic. JAMA Netw Open. 2021;4:e2137395.

35. Toulany A, Kurdyak P, Guttmann A, Stukel TA, Fu L, Strauss R, et al. Trends in the incidence of new-onset anorexia nervosa and atypical anorexia nervosa among youth during the COVID-19 pandemic in Canada. J Adolesc Health. 2022;70:42-47.

36. Dathan-Stumpf A, Vogel M, Hiemisch A, Thiery J, Burkhardt R, Jürgen Kratzsch J, et al. Pediatric reference data of serum lipids and prevalence of dyslipidemia: Results from a population-based cohort in Germany. Clin Biochem. 2016;49:740-9.

37. Song $P$, Zhang $Y, Y u$ J, Zha M, Zhu Y, Rahimi K, et al. Global prevalence of hypertension in children. A systematic review and meta-analysis. JAMA Pediatr. 2019;173:1154-63.

38. Andersen IG, Holm JC, Homøe P. Obstructive sleep apnea in children and adolescents with and without obesity. Eur Arch Otorhinolaryngol. 2019;276:871-8.

39. Anderson EL, Howe LD, Jones HE, Higgins JPT, Lawlor DA, Fraser A. The prevalence of non-alcoholic fatty liver disease in children and adolescents: A systematic review and meta-analysis. PLoS One. 2015;10:e0140908.

\section{ACKNOWLEDGEMENTS}

The authors wish to thank Noga Yaniv and Yeela Bar Tov for assistance with data informatics.

\section{AUTHOR CONTRIBUTIONS}

SS was responsible for substantial contributions to the conception and design of the study, the analysis and interpretation of data, and writing the article. MP was responsible for revising the article critically for important intellectual content. MYG was responsible for the data analysis and interpretation of data, and for revising the article critically for important intellectual content.

\section{COMPETING INTERESTS}

The authors declare no competing interests.

\section{ADDITIONAL INFORMATION}

Supplementary information The online version contains supplementary material available at https://doi.org/10.1038/s41366-022-01092-5.

Correspondence and requests for materials should be addressed to Shlomit Shalitin.

Reprints and permission information is available at http://www.nature.com/ reprints

Publisher's note Springer Nature remains neutral with regard to jurisdictional claims in published maps and institutional affiliations. 